OPEN ACCESS

Edited by:

Dumitru Baleanu,

University of Craiova, Romania

Reviewed by:

Eqab Mahmoud Rabei,

Al al-Bayt University, Jordan

Devendra Kumar

University of Rajasthan, India

Aliyu Isa Aliyu,

Federal University, Dutse, Nigeria

*Correspondence:

Mohammed Al-Refai

m_alrefai@yu.edu.jo

Specialty section:

This article was submitted to

Mathematical Physics,

a section of the journal

Frontiers in Applied Mathematics and

Statistics

Received: 10 February 2018

Accepted: 04 February 2019

Published: 20 February 2019

Citation:

Shat R, Alrefai S, Alhamayda I, Sarhan A and Al-Refai M (2019) The Fractional Laguerre Equation: Series

Solutions and Fractional Laguerre Functions.

Front. Appl. Math. Stat. 5:11. doi: 10.3389/fams.2019.00011

\section{The Fractional Laguerre Equation: Series Solutions and Fractional Laguerre Functions}

\author{
Rasha Shat ${ }^{1}$, Safa Alrefai ${ }^{1}$, Islam Alhamayda ${ }^{1}$, Alaa Sarhan ${ }^{1}$ and Mohammed Al-Refai ${ }^{1,2 *}$ \\ ${ }^{1}$ Department of Mathematical Sciences, United Arab Emirates University, Al Ain, United Arab Emirates, ${ }^{2}$ Department of \\ Mathematics, Yarmouk University, Irbid, Jordan
}

In this paper, we propose a fractional generalization of the well-known Laguerre differential equation. We replace the integer derivative by the conformable derivative of order $0<\alpha<1$. We then apply the Frobenius method with the fractional power series expansion to obtain two linearly independent solutions of the problem. For certain eigenvalues, the infinite series solution truncate to obtain the singular and non-singular fractional Laguerre functions. We obtain the fractional Laguerre functions in closed forms, and establish their orthogonality result. The applicability of the new fractional Laguerre functions is illustrated.

Keywords: fractional differential equations, Laguerre equation, conformable fractional derivative, series solution, Frobenius method

\section{INTRODUCTION}

In recent years, there are interests in studying fractional Sturm-Liouville eigenvalue problems. For instance, the fractional Bessel equation with applications was investigated in Okrasinski and Plociniczak [1, 2], where the fractional derivative is of the Riemann-Liouville type. In Abu Hammad and Khalil [3] the authors solved the fractional Legendre equation with conformable derivative and established the orthogonality property of the fractional Legendre functions. The applications of the fractional Legendre functions in solving fractional differential equations, were illustrated in Kazema et al. [4] and Syam and Al-Refai [5]. In this project we propose the following fractional generalization of the well-known Laguerre differential equation

$$
x^{\alpha} D_{0}^{\alpha} D_{0}^{\alpha} y+\left(1-x^{\alpha}\right) D_{0}^{\alpha} y+\lambda y=0, \frac{1}{2}<\alpha<1, x>0,
$$

where $D_{0}^{\alpha}$ is the conformable derivative of order $\alpha$. The conformable derivative was introduced recently in Khalil et al. [6], and below are the definition and main properties of the derivative.

Definition 1.1. For a function $f:(0, \infty) \rightarrow \mathbb{R}$, the conformable derivative of order $0<\alpha \leq 1$ of $f$ at $x>0$, is defined by

$$
\left(D_{0}^{\alpha} f\right)(x)=\lim _{\epsilon \rightarrow 0} \frac{f\left(x+\epsilon x^{1-\alpha}\right)-f(x)}{\epsilon},
$$

and the derivative at $x=0$ is defined by $\left(D_{0}^{\alpha} f\right)(0)=\lim _{x \rightarrow 0^{+}}\left(D_{0}^{\alpha} f\right)(x)$.

The conformable derivative is a local derivative which has a physical and a geometrical interpretations and potential applications in physics and engineering [7, 8]. It satisfies the nice 
properties of the integer derivative such as, the product rule, the quotient rule, and the chain rule, and it holds that

1. $D_{0}^{\alpha} C=0, C \in \mathbb{R}$,

2. $D_{0}^{\alpha} x^{p}=p x^{p-\alpha}$,

3. $D_{0}^{\alpha} \sin \left(\frac{1}{\alpha} x^{\alpha}\right)=\cos \left(\frac{1}{\alpha} x^{\alpha}\right)$,

4. $D_{0}^{\alpha} \cos \left(\frac{1}{\alpha} x^{\alpha}\right)=-\sin \left(\frac{1}{\alpha} x^{\alpha}\right)$,

5. $D_{0}^{\alpha} e^{\frac{1}{\alpha} x^{\alpha}}=e^{\frac{1}{\alpha} x^{\alpha}}$.

6. $\int_{0}^{a} f(x) d \alpha(x)=\int_{0}^{a} x^{\alpha-1} f(x) d x$.

For more details about the conformable derivative we refer the reader to Abdeljawad [9] and Khalil et al. [6]. We mention here that even though the conformable is a nonlocal derivative (see $[10,11])$, the simplicity and applications of the derivative make it of interests. Also, the applications of the obtained Fractional Leguerre functions are indicated in this manuscript. The rest of the paper is organized as follows: In section 2, we apply the Frobenius method together with the fractional series solution to solve the above equation and to obtain the fractional Laguerre functions. In section 3, we establish the orthogonality result of the fractional Laguerre functions and present the fractional Laguerre functions for several eigenvalues. Finally, we close up with some concluding remarks in section 4 .

\section{THE SERIES SOLUTION}

The series solution is commonly used to solve various types of fractional differential equations (see [12-16]). Since $x=0$, is $\alpha$ regular singular point of Equation (1.1), see [17], we apply the well-known Frobenius method to obtain a solution of the form

$$
y=\sum_{n=0}^{\infty} a_{n} x^{\alpha(n+r)}
$$

where the values of $r$ will be determined. We have

$$
\begin{aligned}
D_{0}^{\alpha} y & =\sum_{n=0}^{\infty} \alpha(n+r) a_{n} x^{\alpha(n+r-1)}, \\
& =\alpha a_{0} r x^{\alpha(r-1)}+\sum_{n=0}^{\infty} \alpha(n+r+1) a_{n+1} x^{\alpha(n+r),} \\
x^{\alpha} D_{0}^{\alpha} y & =\sum_{n=0}^{\infty} \alpha(n+r) a_{n} x^{\alpha(n+r)}, \\
D_{0}^{\alpha} D_{0}^{\alpha} y & =\sum_{n=0}^{\infty} \alpha^{2}(n+r)(n+r-1) a_{n} x^{\alpha(n+r-2)}, \\
x^{\alpha} D_{0}^{\alpha} D_{0}^{\alpha} y & =\sum_{n=0}^{\infty} \alpha^{2}(n+r)(n+r-1) a_{n} x^{\alpha(n+r-1)}, \\
& =\alpha^{2} r(r-1) a_{0} x^{\alpha(r-1)} \\
& +\sum_{n=0}^{\infty} \alpha^{2}(n+r+1)(n+r) a_{n+1} x^{\alpha(n+r)} .
\end{aligned}
$$

By substituting the above results in Equation (1.1) we have

$$
\begin{aligned}
0 & =\alpha^{2} r(r-1) a_{0} x^{\alpha(r-1)}+\sum_{n=0}^{\infty} \alpha^{2}(n+r+1)(n+r) a_{n+1} x^{\alpha(n+r)} \\
& +\alpha a_{0} r x^{\alpha(r-1)}+\sum_{n=0}^{\infty} \alpha(n+r+1) a_{n+1} x^{\alpha(n+r)} \\
& -\sum_{n=0}^{\infty} \alpha(n+r) a_{n} x^{\alpha(n+r)}+\lambda \sum_{n=0}^{\infty} a_{n} x^{\alpha(n+r)} .
\end{aligned}
$$

The coefficients of $x^{\alpha(r-1)}$ will lead to

$$
a_{0} \alpha r(\alpha(r-1)+1)=0 .
$$

Because $\alpha \neq 0$, and $a_{0}=0$, will lead to the zero solution, we have

$$
r=0, r=1-\frac{1}{\alpha} .
$$

We start with $r=0$, we have

$$
\alpha^{2} n(n+1) a_{n+1}+\alpha(n+1) a_{n+1}-\alpha n a_{n}+\lambda a_{n}=0,
$$

or

$$
a_{n+1}=\frac{\alpha n-\lambda}{\alpha(n+1)(\alpha n+1)} a_{n}, \quad n \geq 0 .
$$

Lemma 2.1. The coefficients $a_{n}$ in Equation (2.3) satisfy

$$
a_{n+1}=\frac{\prod_{j=0}^{n}(j \alpha-\lambda)}{\alpha^{n+1}(n+1) ! \prod_{j=0}^{n}(j \alpha+1)} a_{0}, \quad n \geq 0 .
$$

Proof: The proof can be easily obtained by iterating the recursion in (2.3) and applying induction arguments.

Remark 2.1. For $\alpha=1$, the recursion relation in (2.4) will reduce to

$$
a_{n+1}=\frac{-\lambda(1-\lambda)(2-\lambda) \cdots(n-\lambda)}{[(n+1) !]^{2}} a_{0},
$$

which is exactly the recursion relation that has been obtained in solving the Laguerre equation with integer derivative.

For $r=-\frac{1}{\alpha}+1$, we have for $n \geq 0$,

$$
\begin{aligned}
a_{n+1} & =\frac{\alpha(n+r)-\lambda}{\alpha(n+r+1)(\alpha[n+r]+1)} a_{n}, \\
& =\frac{\alpha(n+1)-(\lambda+1)}{\alpha(n+1)(\alpha[n+2]-1)} a_{n} .
\end{aligned}
$$

By iterating the recursion in (2.6) and applying induction arguments, we have 
Lemma 2.2. The coefficients $a_{n}$ in Equation (2.6) satisfy

$$
a_{n+1}=\frac{\prod_{j=1}^{n+1}(j \alpha-[\lambda+1])}{\alpha^{n+1}(n+1) ! \prod_{j=2}^{n+2}(j \alpha-1)} a_{0}, \quad n \geq 0 .
$$

Remark 2.2. By applying the Frobenius method to the regular Laguerre equation with integer derivative $\alpha=1$, we obtain only one value of $r=0$, which produces only one solution. Here with the fractional case, we obtain two values of $r=0,1-\frac{1}{\alpha}$, that will produce two linearly independent solutions of the problem as we will see later.

Now, in Equation (2.4), if we choose $\alpha=\alpha_{m}$ and $\lambda=\lambda_{m}$ such that

$$
m \alpha_{m}=\lambda_{m}
$$

for some integer $m$, then

$$
a_{m+1}=a_{m+2}=\cdots=0,
$$

and the infinite series solution will truncate to obtain the finite sum

$$
\begin{aligned}
u(x) & =\sum_{n=0}^{m} a_{n} x^{n \alpha_{m}}=a_{0}\left(1+\sum_{n=1}^{m} \frac{\prod_{j=0}^{n-1}\left(j \alpha_{m}-\lambda_{m}\right)}{\alpha_{m}^{n} n ! \prod_{j=0}^{n-1}\left(j \alpha_{m}+1\right)} x^{n \alpha_{m}}\right) \\
& =a_{0} L_{m, \alpha_{m}}^{0}(x),
\end{aligned}
$$

where $L_{m, \alpha_{m}}^{0}(x)$ is the non-singular fractional Laguerre function of order $m$. Since

$$
\begin{aligned}
\prod_{j=0}^{n-1}\left(j \alpha_{m}-\lambda_{m}\right) & =\prod_{j=0}^{n-1}\left(j \alpha_{m}-m \alpha_{m}\right)=\prod_{j=0}^{n-1} \alpha_{m} \prod_{j=0}^{n-1}(j-m) \\
& =\alpha_{m}^{n} \prod_{j=0}^{n-1}(j-m),
\end{aligned}
$$

then

$$
L_{m, \alpha_{m}}^{0}(x)=1+\sum_{n=1}^{m} \frac{\prod_{j=0}^{n-1}(j-m)}{n ! \prod_{j=0}^{n-1}\left(j \alpha_{m}+1\right)} x^{n \alpha_{m}} .
$$

Analogously, in Equation (2.7), if we choose $\alpha=\alpha_{m}$ and $\lambda=\lambda_{m}$ such that

$$
m \alpha_{m}=\lambda_{m}+1
$$

then

$$
a_{m}=a_{m+1}=\cdots=0 \text {, }
$$

and the infinite series solution will truncate to obtain the solution

$$
\begin{aligned}
u(x) & =\sum_{n=0}^{m-1} a_{n} x^{\alpha_{m}\left(n-\frac{1}{\alpha_{m}}+1\right)}=x^{-1} \sum_{n=0}^{m-1} a_{n} x^{\alpha_{m}(n+1)} \\
& =a_{0} L_{m-1, \alpha_{m}}^{1}(x)
\end{aligned}
$$

where

$$
\begin{aligned}
L_{m-1, \alpha_{m}}^{1}(x) & =x^{-1}\left(x_{m}^{\alpha}+\sum_{n=1}^{m-1} \frac{\prod_{j=1}^{n}\left(j \alpha_{m}-\left(\lambda_{m}+1\right)\right)}{\alpha_{m}^{n} n ! \prod_{j=2}^{n+1}\left(j \alpha_{m}-1\right)} x^{\alpha_{m}(n+1)}\right) \\
& =x^{\alpha_{m}-1}\left(1+\sum_{n=1}^{m-1} \frac{\prod_{j=1}^{n}\left(j \alpha_{m}-m \alpha_{m}\right)}{\alpha_{m}^{n} n ! \prod_{j=2}^{n+1}\left(j \alpha_{m}-1\right)} x^{\alpha_{m} n}\right) \\
& =x^{\alpha_{m}-1}\left(1+\sum_{n=1}^{m-1} \frac{\prod_{j=1}^{n}(j-m)}{n ! \prod_{j=2}^{n+1}\left(j \alpha_{m}-1\right)} x^{\alpha_{m} n}\right)
\end{aligned}
$$

is the fractional singular Laguerre function of order $m-1$.

Remark 2.3. If we substitute $\alpha_{m}=1$, then

$$
L_{m, 1}^{0}(x)=L_{m, 1}^{1}(x)=1+\sum_{n=1}^{m} \frac{\prod_{j=0}^{n-1}(j-m)}{n ! \prod_{j=0}^{n-1}(j+1)} .
$$

Since $\prod_{j=0}^{n-1}(j+1)=n !$, and $\prod_{j=0}^{n-1}(j-m)=(-1)^{n} \frac{m !}{(m-n) !}$, we have

$$
L_{m, 1}^{0}(x)=L_{m, 1}^{1}(x)=1+\sum_{n=1}^{m} \frac{(-1)^{m} m !}{(n !)^{2}(m-n) !}
$$

which is the expansion of the Laguerre polynomial $L_{m}(x)$.

\section{THE FRACTIONAL LAGUERRE FUNCTIONS}

We start with the orthogonality property of the fractional Laguerre functions $\left(L_{m, \alpha_{m}}(x)\right), m=0,1,2, \cdots$. Here by $L_{m, \alpha_{m}}(x)$ we mean the non-singular and singular Laguerre functions obtained in (2.8) and (2.9).

Theorem 3.1. The fractional Laguerre functions $\left(L_{m, \alpha_{m}}(x)\right), m=$ $0,1,2, \cdots$ are orthogonal on $(0, \infty)$ with respect to the weight function $\mu(x)=e^{-\frac{x^{\alpha}}{\alpha}}$,i.e.,

$$
\int_{0}^{\infty} e^{-\frac{x^{\alpha}}{\alpha}} L_{m, \alpha_{m}}(x) L_{n, \alpha_{n}}(x) d x=0, m \neq n
$$


Proof: One can easily prove that Equation (1.1) can be written as

$$
D_{0}^{\alpha}\left(x e^{-\frac{x^{\alpha}}{\alpha}} D_{0}^{\alpha} y\right)=-\lambda x^{1-\alpha} e^{-\frac{x^{\alpha}}{\alpha}} y
$$

Thus, the equation is of a special type of the fractional Sturmliouville eigenvalue problem

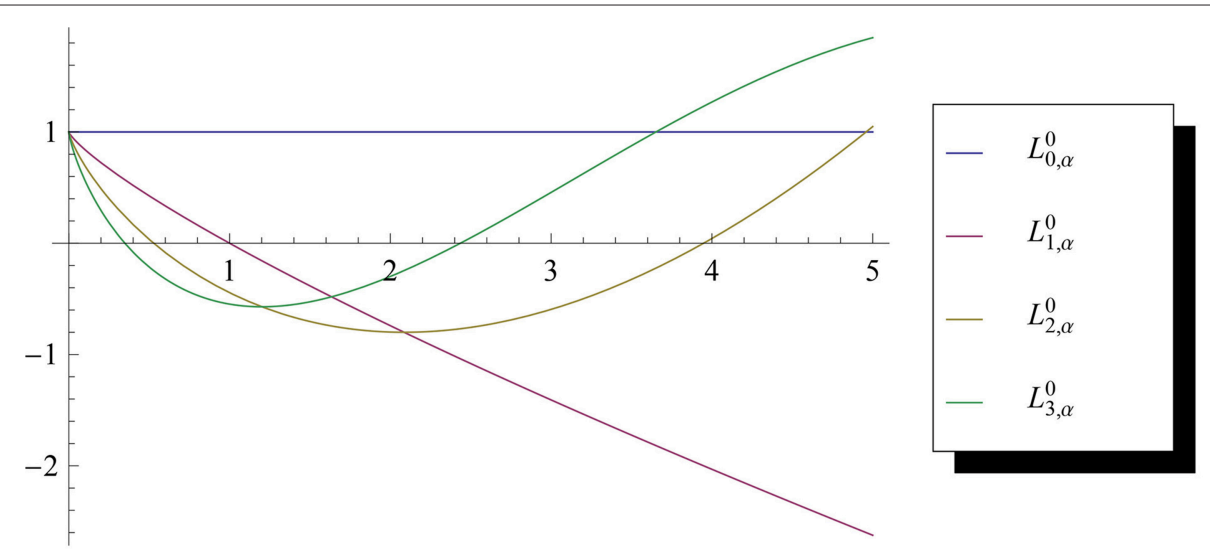

FIGURE 1 | A plot of $L_{0, \alpha}^{0}, L_{1, \alpha}^{0}, L_{2, \alpha}^{0}, L_{3, \alpha}^{0}$ for $\alpha=0.8$.

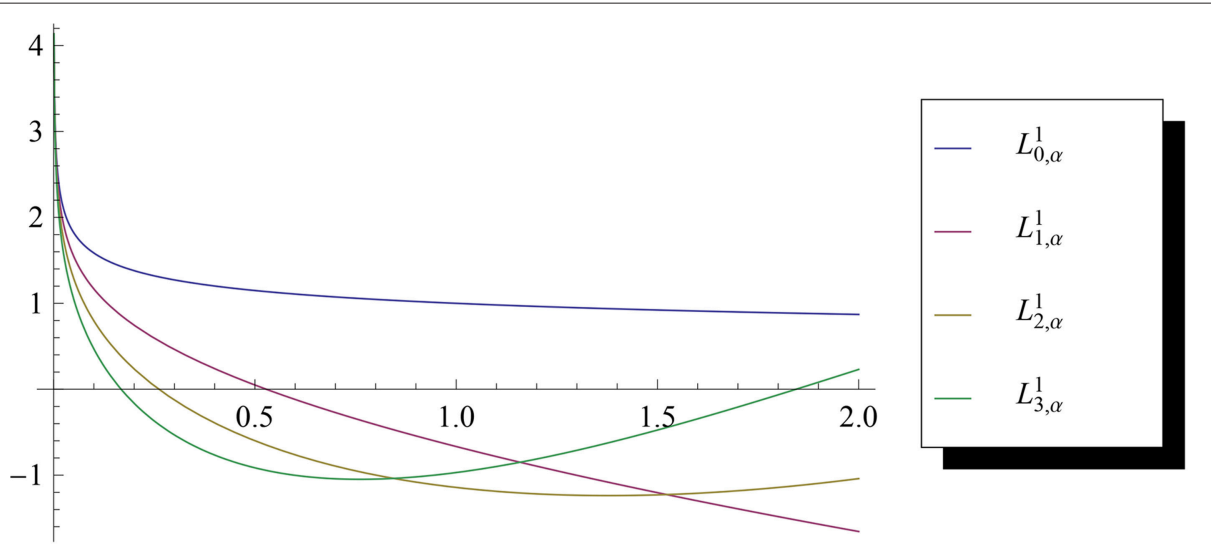

FIGURE 2 | A plot of $L_{0, \alpha}^{1}, L_{1, \alpha}^{1}, L_{2, \alpha}^{1}, L_{3, \alpha}^{1}$ for $\alpha=0.8$

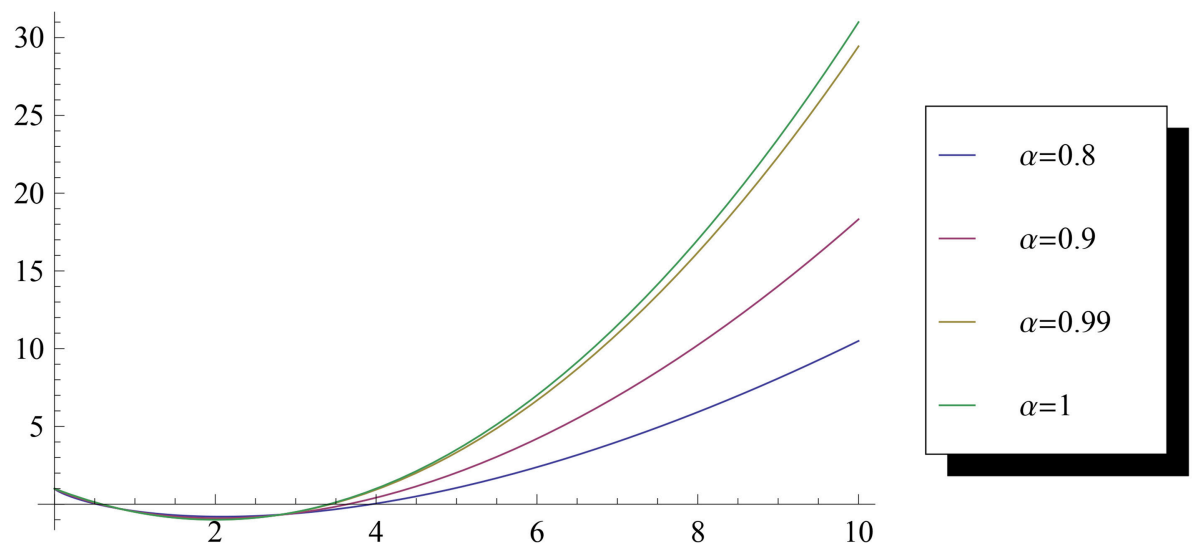

FIGURE 3 | A plot of $L_{2, \alpha}^{0}$, for $\alpha=0.8,0.9,0.99,1$. 
where $p(x)=x e^{-\frac{x^{\alpha}}{\alpha}}, q(x)=0$ and $w(x)=x^{1-\alpha} e^{-\frac{x^{\alpha}}{\alpha}}$. Using the fractional Lagrange Identity obtained in Al-Refai and Abdeljawad [18], we have

$$
\begin{aligned}
& -\left(\lambda_{m}-\lambda_{n}\right) \int_{0}^{\infty} w(x) L_{m, \alpha_{m}} L_{n, \alpha_{n}} d \alpha(x) \\
& =\left.p(x)\left(L_{n, \alpha_{n}} D_{0}^{\alpha} L_{m, \alpha_{m}}-L_{m, \alpha_{m}} D_{0}^{\alpha} L_{n, \alpha_{n}}\right)\right|_{0} ^{\infty} .
\end{aligned}
$$

We have $p(0)=0$, and

$$
\lim _{x \rightarrow \infty} x^{1-\alpha} e^{-\frac{x^{\alpha}}{\alpha}}\left(L_{n, \alpha_{n}} D_{0}^{\alpha} L_{m, \alpha_{m}}-L_{m, \alpha_{m}} D_{0}^{\alpha} L_{n, \alpha_{n}}\right)(x)=0 .
$$

Thus the right hand side of Equation (3.2) equals zero which together with $\lambda_{m} \neq \lambda_{n}$ will lead to

$$
\begin{aligned}
\int_{0}^{\infty} w(x) L_{m, \alpha_{m}} L_{n, \alpha_{n}} d \alpha(x) & =\int_{0}^{\infty} x^{1-\alpha} e^{-\frac{x^{\alpha}}{\alpha}} L_{m, \alpha_{m}}(x) L_{n, \alpha_{n}}(x) x^{\alpha-1} d x \\
& =\int_{0}^{\infty} e^{-\frac{x^{\alpha}}{\alpha}} L_{m, \alpha_{m}}(x) L_{n, \alpha_{n}}(x) d x=0
\end{aligned}
$$

and hence the result.

Remark 3.1. Since the fractional Laguerre functions are orthogonal, they can be used as a basis of the spectral method to study fractional differential equations analytically and numerically. They also can be used as a basis of the fractional Gauss-Laguerre quadrature for approximating the value of integrals of the form

$$
\int_{0}^{\infty} e^{-\frac{x^{\alpha}}{\alpha}} f(x) d x
$$

Remark 3.2. New types of improper integrals are determined using the orthogonality property which are not known before, such as

$$
\begin{aligned}
& \int_{0}^{\infty}\left(1-x^{\alpha}\right) e^{-\frac{x^{\alpha}}{\alpha}} d x=0, L_{0, \alpha_{0}}^{0}(x)=1, L_{1, \alpha_{1}}^{0}(x)=1-x^{\alpha_{1}}, \\
& \int_{0}^{\infty} x^{2(\alpha-1)}\left(1-\frac{1}{2 \alpha-1} x^{\alpha}\right) e^{-\frac{x^{\alpha}}{\alpha}} d x=0 \\
& L_{0, \alpha_{0}}^{1}(x)=x^{\alpha_{0}-1}, L_{1, \alpha_{1}}^{1}(x)=x^{\alpha_{1}-1}\left(1-\frac{1}{2 \alpha_{1}-1} x^{\alpha_{1}}\right),
\end{aligned}
$$

In the following we present the singular and non-singular fractional Laguerre functions of several orders.

$$
L_{0, \alpha_{0}}^{0}(x)=1
$$

\section{REFERENCES}

1. Okrasinski W, Plociniczak L. A note of fractional Bessel equation and its asymptotics. Fract Calc Appl Anal. (2013) 16:559-72. doi: 10.2478/s13540-013-0036-5

$$
\begin{aligned}
L_{1, \alpha_{1}}^{0}(x) & =1-x^{\alpha_{1}} \\
L_{2, \alpha_{2}}^{0}(x) & =1-2 x^{\alpha_{2}}+\frac{1}{\alpha_{2}+1} x^{2 \alpha_{2}} \\
L_{3, \alpha_{3}}^{0}(x) & =1-3 x^{\alpha_{3}}+\frac{3}{\alpha_{3}+1} x^{2 \alpha_{3}}-\frac{1}{\left(\alpha_{3}+1\right)\left(2 \alpha_{3}+1\right)} x^{3 \alpha_{3}} . \\
L_{0, \alpha_{0}}^{1}(x) & =x^{\alpha_{0}-1} \\
L_{1, \alpha_{1}}^{1}(x) & =x^{\alpha_{1}-1}\left(1-\frac{1}{2 \alpha_{1}-1} x^{\alpha_{1}}\right), \\
L_{2, \alpha_{2}}^{1}(x) & =x^{\alpha_{2}-1}\left(1-\frac{2}{2 \alpha_{2}-1} x^{\alpha_{2}}+\frac{1}{\left(2 \alpha_{2}-1\right)\left(3 \alpha_{2}-1\right)} x^{2 \alpha_{2}}\right), \\
L_{3, \alpha_{3}}^{1}(x) & =x^{\alpha_{3}-1}\left(1-\frac{3}{2 \alpha_{3}-1} x^{\alpha_{3}}+\frac{3}{\left(2 \alpha_{3}-1\right)\left(3 \alpha_{3}-1\right)} x^{2 \alpha_{3}}\right. \\
& \left.-\frac{1}{\left(2 \alpha_{3}-1\right)\left(3 \alpha_{3}-1\right)\left(4 \alpha_{3}-1\right)} x^{3 \alpha_{3}}\right) .
\end{aligned}
$$

Figures 1, 2 depict the non-singular and singular fractional Laguerre functions of several orders for $\alpha=0.8$. Figure 3 depicts $L_{2, \alpha}^{0}$ for several values of $\alpha$. One can see that, as $\alpha$ approaches 1 , the non-singular fractional Laguerre functions approach the Laguerre polynomial of degree 2 .

\section{CONCLUSION}

We have considered the fractional Laguerre equation with conformable derivative. We obtained two linearly independent solutions using the fractional series solution and Frobenius method. The first non-singular solution is analytic on $(0, \infty)$, and the second singular solution has a singularity at $x=0$. For certain eigenvalues, these infinite solutions truncate to obtain the fractional Laguerre functions. Because of the orthogonality property of the fractional Laguerre functions, they can be used as a basis of the spectral method to study fractional differential equations, or as a basis of the Gauss-Laguerre quadrature for evaluating certain integrals. The obtained results coincide with the ones of the regular Laguerre polynomials as the derivative $\alpha$ approaches 1 .

\section{AUTHOR CONTRIBUTIONS}

All authors listed have made a substantial, direct and intellectual contribution to the work, and approved it for publication.

\section{ACKNOWLEDGMENTS}

The authors gratefully acknowledge the support of the United Arab Emirates University under the SURE+ grant no. 31S291_26_2_ SURE+2017.

2. Okrasinski W, Plociniczak L. On fractional Bessel equation and the description of corneal topography. arXiv.1201.2526v2 [math.CA] (2012).

3. Abu Hammad M, Khalil R. Legendre fractional differential equation and Legender fractional polynomials. Int J Appl Math Res. (2014) 3: 214-9. doi: 10.14419/ijamr.v3i3.2747 
4. Kazema S, Abbasbandya S, Kumarb S. Fractional-order Legendre functions for solving fractional-order differential equations. Appl Math Model. (2013) 37:5498-510. doi: 10.1016/j.apm.2012.10.026

5. Syam M, Al-Refai M. Solving fractional diffusion equation via the collocation method based on fractional Legendre functions. J Comput Methods Phys. (2014) 2014:381074. doi: 10.1155/2014/381074

6. Khalil $\mathrm{R}$, Al Horani M, Yousef A, Sababheh M. A new definition of fractional derivative. J Comput Appl Math. (2015) 264:65-70. doi: 10.1016/j.cam.2014.01.002

7. Usta F, sarikaya M. Some improvements of conformable fractional integral inequalities. Int J Anal Appl. (2017) 14:162-6.

8. Zhao D, Luo M. General conformable fractional derivative and its physical interpretation. Calcolo (2017) 54:903-17. doi: 10.1007/s10092-017-0213-8

9. Abdeljawad T. On conformable fractional calculus. J Comput Appl Math (2015) 279:57-66. doi: 10.1016/j.cam.2014.10.016

10. Liu CS. On the local fractional derivative of everywhere non-differentiable continuous functions on intervals. Commun Nonlin Sci Numer Simul. (2017) 42:229-35. doi: 10.1016/j.cnsns.2016.05.029

11. Tarasov VE. No nonlocality. No fractional derivative. Commun Nonlin Sci Numer Simul. (2018) 62:157-63. doi: 10.1016/j.cnsns.2018.02.019

12. Al-Refai M, Hajji M, Syam M. An efficient series solution for fractional differential equations. Abstr Appl Anal. (2014) 2014:891837. doi: $10.1155 / 2014 / 891837$

13. Al-Refai M, Syam M, Al-Mdallal Q. On the fractional Legendre equation and fractional Legendre functions. Progr Fract Differ Appl. (2017) 3:93-102. doi: 10.18576/pfda/030202

14. Baleanu D, Inc M, Yusuf A, Aliyo A. Space-time fractional RosenouHaynam equation: lie symmetry analysis, explicit solutions and conservation laws. Adv Differ Equat. (2018) 2018:64. doi: 10.1186/s13662-0181468-3

15. Baleanu D, Inc M, Yusuf A, Aliyo A. Lie symmetry analysis, exact solutions and conservation laws for the time fractional Caudrey-Dodd-GibbonSawada-Kotera equation. Commun Nonlin Sci Numer Simul. (2018) 59:22234. doi: 10.1016/j.cnsns.2017.11.015

16. Inc M, Yusuf A, Aliyo A, Baleanu D. Lie symmetry analysis, explicit solutions and conservation laws for the space-time fractional nonlinear evolution equations. Phys A (2018) 496:371-83. doi: 10.1016/j.physa.2017. 12.119

17. Unal E, Gokdogan A, Celik E. Solutions around $\alpha$ singular point of a sequential conformable fractional differential equation. Kuwait J Sci. (2017) 44:9-16.

18. Al-Refai M, Abdeljawad T. Fundamental results of conformable Sturm-Liouville eigenvalue problems. Complexity (2017) 2017:3720471. doi: $10.1155 / 2017 / 3720471$

Conflict of Interest Statement: The authors declare that the research was conducted in the absence of any commercial or financial relationships that could be construed as a potential conflict of interest.

Copyright (c) 2019 Shat, Alrefai, Alhamayda, Sarhan and Al-Refai. This is an openaccess article distributed under the terms of the Creative Commons Attribution License (CC BY). The use, distribution or reproduction in other forums is permitted, provided the original author(s) and the copyright owner(s) are credited and that the original publication in this journal is cited, in accordance with accepted academic practice. No use, distribution or reproduction is permitted which does not comply with these terms. 